\title{
Partial Variable Stability for a Class of Nonlinear Systems with Time Delay
}

\author{
Ran Huo', Xiaoli Wang2* \\ ${ }^{1}$ College of Science, Inner Mongolia Agricultural University, Huhhot, China \\ ${ }^{2}$ College of Statistics and Mathematics, Inner Mongolia University of Finance and Economics, Huhhot, China \\ Email: huoran124@163.com, ^ncdwangxiaoli@163.com
}

How to cite this paper: Huo, R. and Wang, X.L. (2020) Partial Variable Stability for a Class of Nonlinear Systems with Time Delay. Applied Mathematics, 11, 377-388. https://doi.org/10.4236/am.2020.115027

Received: April 7, 2020

Accepted: May 10, 2020

Published: May 13, 2020

Copyright (c) 2020 by author(s) and Scientific Research Publishing Inc. This work is licensed under the Creative Commons Attribution International License (CC BY 4.0).

http://creativecommons.org/licenses/by/4.0/

\begin{abstract}
This article first gives a new class of integral inequalities. Then, as an application, the nonlinear neutral differential system with multiple delays is considered, and the trivial solution of the nonlinear neutral system with multiple delays is obtained. Uniform asymptotic Lipschitz stability. Obviously, the above system is a generalization of the traditional differential system. The purpose of this paper is to study the dual stability of neutral differential equations with delays, including equal asymptotically Lipschitz stability and uniformly asymptotic Lipschitz stability. The author uses the method of integral inequality to establish a double stability criterion. As a result, the local stability of differential equations is widely used in theory and practice, such as dynamic systems and control systems.
\end{abstract}

\section{Keywords}

Nonlinear Neutral Systems, Double Stability, Lipschitz Asymptotic Stability, Integral Inequality

\section{Introduction}

In 1892, Lyapunov, a Russian mathematician, mechanician and physicist, proposed the notion of the stability of motion. He gave the general research methods in his doctoral dissertation "The general problem of the stability of motion" [1], in which he established the foundation of the stability theory. When studying nonlinear systems, especially studying dynamic systems or control systems, we cannot study the stability of all variables because of the technology difficulties, the limitation of practical conditions, or it is not necessary to study all variables considering the actual need. As a result, studying the partial stability of differential equations becomes more important. In addition, the partial stability is widely 
used in science and technology. For instance the absolute stability of famous Lurie adjusting systems can be changed into a problem of partial stability. In a word, it is of practical significance to study the partial stability of differential equations.

Since Bellman created a class of integral inequalities in 1958, integral inequalities have been greatly developed. The main results are:

In 1960, Li Yuesheng gave the following inequality in [2]:

$$
u(t) \leq u_{0}+\int_{0}^{t} g(s) u(s) \mathrm{d} s+\int_{0}^{t} f(s) u^{\alpha}(s) \mathrm{d} s
$$

In 2005, Sligeng discussed the following inequality in [3]:

$$
\begin{aligned}
u_{1}(t) \leq & k_{1}+\int_{0}^{t} h_{1}(s) u_{1}(s) \mathrm{d} s+\int_{0}^{t} h_{2}(s) u_{2}(s) \mathrm{e}^{\mu s} \mathrm{~d} s \\
+ & \int_{0}^{t} \bar{h}_{1}(s) u_{1}^{\alpha}(s) \mathrm{e}^{-(\alpha-1) \mu s} \mathrm{~d} s+\int_{0}^{t} \bar{h}_{2}(s) u_{2}^{\alpha}(s) \mathrm{e}^{\mu s} \mathrm{~d} s \\
u_{2}(t) \leq & k_{2}+\int_{0}^{t} h_{3}(s) u_{1}(s) \mathrm{e}^{-\mu s} \mathrm{~d} s+\int_{0}^{t} h_{4}(s) u_{2}(s) \mathrm{d} s \\
& +\int_{0}^{t} \bar{h}_{3}(s) u_{1}^{\alpha}(s) \mathrm{e}^{-\alpha \mu s} \mathrm{~d} s+\int_{0}^{t} \bar{h}_{4}(s) u_{2}^{\alpha}(s) \mathrm{d} s
\end{aligned}
$$

In 2009, the author discussed a new class of inequalities in [4].

Vorotnikov, V.I. [5] [6] considered the following system

$$
\left\{\begin{array}{l}
\frac{\mathrm{d} y}{\mathrm{~d} t}=A(t) y+B(t) z+Y(t, y, z) \\
\frac{\mathrm{d} z}{\mathrm{~d} t}=C(t) y+D(t) z+Z(t, y, z)
\end{array}\right.
$$

and studied the double stability as $\|y\|+\|z\| \rightarrow 0$ and $\frac{\|Y(t, y, z)\|+\|Z(t, y, z)\|}{\|y\|+\|z\|} \rightarrow 0$.

In 2002, Wang Feng used the differential inequality of delay in article [7] to study the following delay system:

$$
\left\{\begin{array}{l}
\frac{\mathrm{d} y}{\mathrm{~d} t}=A(t) y+B(t) z+Y(t, y, z, y(t-\tau), z(t-\tau)) \\
\frac{\mathrm{d} z}{\mathrm{~d} t}=C(t) y+D(t) z+Z(t, y, z, y(t-\tau), z(t-\tau))
\end{array}\right.
$$

In 2006, Siligeng used the integral inequality extended in [3] in [8] to discuss the double stability of the following system to some variables:

$$
\left\{\begin{array}{l}
\frac{\mathrm{d} y}{\mathrm{~d} t}=A(t) y+f_{1}(t, y, z) \\
\frac{\mathrm{d} z}{\mathrm{~d} t}=B(t) z+f_{2}(t, y, z)
\end{array}\right.
$$

In this paper the author consider a new class of the nonlinearly perturbed differential systems with time-delay

$$
\left\{\begin{array}{l}
\frac{\mathrm{d} y}{\mathrm{~d} t}=B(t) y+C(t) z+Y\left(s, y(s), z(s), \int_{0}^{t} h_{1}(s, y(s), z(s), y(s-\tau), \dot{z}(s-\tau)) \mathrm{d} s\right) \\
\frac{\mathrm{d} z}{\mathrm{~d} t}=D(t) y+E(t) z+Z\left(s, y(s), z(s), \int_{0}^{t} h_{2}(s, y(s), z(s), y(s-\tau), \dot{z}(s-\tau)) \mathrm{d} s\right)
\end{array}\right.
$$


It is obvious that the above system is a generalization of the systems in [5] [6] [7] [8].

The aim of this paper is to investigate the double stability of neutural differential equations, including Uniform stability and Uniform Lipschitz stability. The author uses the method of differential inequalities with time-delay and integral inequalities to establish double stability criteria.

\section{Preliminaries}

Consider the following system:

$$
\frac{\mathrm{d} x}{\mathrm{~d} t}=f(t, x(t), x(t-\tau), \dot{x}(t-\tau))
$$

where $x \in R^{n}, \quad y=\operatorname{col}\left(x_{1}, x_{2}, \cdots, x_{m}\right), \quad z=\operatorname{col}\left(x_{m+1}, x_{m+2}, \cdots, x_{n}\right), \quad x=\operatorname{col}(y, z)$, $f(t, 0,0) \equiv 0, \tau$ is a nonnegative constant. Let $\phi(t)$ be a continuous function, for $\forall t \in E_{t_{0}}=\left[t_{0}-\tau, t_{0}\right]$.

Definition 1 [9] [10] [11] The trivial solution of system (1) has uniform stability and exponential asymptotic stability with respect to $y$ if, for $\forall \varepsilon>0$, $\forall t_{0} \in I, \exists \delta(\varepsilon)>0$, and $\lambda>0$, when $\|\phi\|<\delta$ (for $\forall t \in E_{t_{0}}$ ), such that

$$
\left\|y\left(t ; t_{0}, \phi\right)\right\|+\left\|\dot{y}\left(t ; t_{0}, \phi\right)\right\|<\varepsilon \exp \left(-\lambda\left(t-t_{0}\right)\right), \forall t \geq t_{0} .
$$

Definition 2 [9] [10] [11] The trivial solution of system (1) has Lipschitz stability with respect to $y$ if, there exists constants $M\left(t_{0}\right)>0$ and $\delta\left(t_{0}\right)>0$, when $\|\varphi\|+\|\dot{\varphi}\|<\delta \quad\left(\right.$ for $\left.\forall t \in E_{t_{0}}\right)$, such that

$$
\left\|y\left(t ; t_{0}, \phi\right)\right\|+\left\|\dot{y}\left(t ; t_{0}, \phi\right)\right\| \leq M\left(t_{0}\right)(\|\phi\|+\|\dot{\phi}\|), \forall t \geq t_{0} \geq 0 .
$$

Definition 3 [9] [10] [11] The trivial solution of system (1) has equi-exponential Lipschitz asymptotic stability with respect to $y$ if, there exists $\lambda>0, K\left(t_{0}\right)>0$ and $\delta\left(t_{0}\right)>0$, when $\|\varphi\|+\|\dot{\varphi}\|<\delta$ (for $\forall t \in E_{t_{0}}$ ), such that

$$
\left\|y\left(t ; t_{0}, \phi\right)\right\|+\left\|\dot{y}\left(t ; t_{0}, \phi\right)\right\| \leq K\left(t_{0}\right)(\|\phi\|+\|\dot{\phi}\|) \exp \left(-\lambda\left(t-t_{0}\right)\right), \forall t \geq t_{0} \geq 0 .
$$

Definition 4 [9] [10] [11] The trivial solution of system (1) has uniform exponential Lipschitz asymptotic stability with respect to $y$ if, $K$ and $\delta>0$ in definition 3 are ndependent of $t_{0}$.

Lemma 1 [4] The following conditions are established on $t \geq t_{0}$ :

i) $k_{1}, k_{2}, \mu$ are non-negative constants;

ii) 1)

$$
\begin{aligned}
u_{1}(t) \leq & k_{1}+\int_{t_{0}}^{t} a(s) u_{1}(s) \mathrm{d} s+\int_{t_{0}}^{t} b(s) u_{2}(s) \mathrm{e}^{\mu\left(s-t_{0}\right)} \mathrm{d} s \\
& +\int_{t_{0}}^{t} \sum_{i=1}^{I} c_{i}(s) u_{1}^{\alpha_{i}+1}(s) \mathrm{e}^{-\alpha_{i} \mu\left(s-t_{0}\right)} \mathrm{d} s+\int_{t_{0}}^{t} \sum_{i=1}^{I} d_{i}(s) u_{2}^{\alpha_{i}+1}(s) \mathrm{e}^{\mu\left(s-t_{0}\right)} \mathrm{d} s \\
& +\int_{t_{0}}^{t} \sum_{j=1}^{J} e_{j}(s) \int_{t_{0}}^{s} f_{j}(\tau) u_{1}(\tau) \mathrm{d} \tau \mathrm{d} s \\
& +\int_{t_{0}}^{t} \sum_{j=1}^{J} g_{j}(s)\left[\int_{t_{0}}^{s} h_{j}(\tau) u_{2}(\tau) \mathrm{e}^{\mu\left(\tau-t_{0}\right)} \mathrm{d} \tau\right] \mathrm{d} s \\
& +\int_{t_{0}}^{t} \sum_{k=1}^{K} o_{k}(s) \int_{t_{0}}^{s} p_{k}(\tau) u_{1}^{\beta_{k}+1}(\tau) \mathrm{e}^{-\beta_{k} \mu\left(\tau-t_{0}\right)} \mathrm{d} \tau \mathrm{d} s
\end{aligned}
$$




$$
\begin{aligned}
& +\int_{t_{0}}^{t} \sum_{k=1}^{K} q_{k}(s) \int_{t_{0}}^{s} r_{k}(\tau) u_{2}^{\beta_{k}+1}(\tau) \mathrm{e}^{\mu\left(\tau-t_{0}\right)} \mathrm{d} \tau \mathrm{d} s \\
& +\int_{t_{0}}^{t} \sum_{i=1}^{I} c_{i}(s) u_{1}^{\alpha_{i}}(s) \mathrm{e}^{-\alpha_{i} \mu\left(s-t_{0}\right)} \int_{t_{0}}^{s} \sum_{l=1}^{L} v_{l}(\theta) \int_{t_{0}}^{\theta} w_{l}(\tau) u_{1}(\tau) \mathrm{d} \tau \mathrm{d} \theta \mathrm{d} s \\
& +\int_{t_{0}}^{t} \sum_{i=1}^{I} d_{i}(s) u_{2}^{\alpha_{i}}(s) \int_{t_{0}}^{s} \sum_{l=1}^{L} x_{l}(\theta) \int_{t_{0}}^{\theta} y_{l}(\tau) u_{2}(\tau) \mathrm{e}^{\mu\left(\tau-t_{0}\right)} \mathrm{d} \tau \mathrm{d} \theta \mathrm{d} s \\
& +\int_{t_{0}}^{t} \sum_{i=1}^{I} c_{i}(s) u_{1}^{\alpha_{i}}(s) \mathrm{e}^{-\alpha_{i} \mu\left(s-t_{0}\right)} \int_{t_{0}}^{s} \sum_{m=1}^{M} A_{m}(\theta) \int_{t_{0}}^{\theta} B_{m}(\tau) u_{1}^{\gamma_{m}+1}(\tau) \mathrm{e}^{-\gamma_{m} \mu\left(\tau-t_{0}\right)} \mathrm{d} \tau \mathrm{d} \theta \mathrm{d} s \\
& +\int_{t_{0}}^{t} \sum_{i=1}^{I} d_{i}(s) u_{2}^{\alpha_{i}}(s) \int_{t_{0}}^{s} \sum_{m=1}^{M} D_{m}(\theta) \int_{t_{0}}^{\theta} E_{m}(\tau) u_{2}^{\gamma_{m}+1}(\tau) \mathrm{e}^{\mu\left(\tau-t_{0}\right)} \mathrm{d} \tau \mathrm{d} \theta \mathrm{d} s
\end{aligned}
$$

2)

$$
\begin{aligned}
& u_{2}(t) \leq k_{2}+\int_{t_{0}}^{t} \bar{a}(s) u_{1}(s) \mathrm{e}^{-\mu\left(s-t_{0}\right)} \mathrm{d} s+\int_{t_{0}}^{t} \bar{b}(s) u_{2}(s) \mathrm{d} s \\
& +\int_{t_{0}}^{t} \sum_{i=1}^{I} \bar{c}_{i}(s) u_{1}^{\alpha_{i}+1}(s) \mathrm{e}^{-\left(\alpha_{i}+1\right) \mu\left(s-t_{0}\right)} \mathrm{d} s+\int_{t_{0}}^{t} \sum_{i=1}^{I} \bar{d}_{i}(s) u_{2}^{\alpha_{i}+1}(s) \mathrm{d} s \\
& +\int_{t_{0}}^{t} \sum_{j=1}^{J} \bar{e}_{j}(s) \int_{t_{0}}^{s} \bar{f}_{j}(\tau) u_{1}(\tau) \mathrm{e}^{-\mu\left(\tau-t_{0}\right)} \mathrm{d} \tau \mathrm{d} s \\
& +\int_{t_{0}}^{t} \sum_{j=1}^{J} \bar{g}_{j}(s)\left[\int_{t_{0}}^{s} \bar{h}_{j}(\tau) u_{2}(\tau) \mathrm{d} \tau\right] \mathrm{d} s \\
& +\int_{t_{0}}^{t} \sum_{k=1}^{K} \bar{o}_{k}(s) \int_{t_{0}}^{s} \bar{p}_{k}(\tau) u_{1}^{\beta_{k}+1}(\tau) \mathrm{e}^{-\left(\beta_{k}+1\right) \mu\left(\tau-t_{0}\right)} \mathrm{d} \tau \mathrm{d} s \\
& +\int_{t_{0}}^{t} \sum_{k=1}^{K} \bar{q}_{k}(s) \int_{t_{0}}^{s} \bar{r}_{k}(\tau) u_{2}^{\beta_{k}+1}(\tau) \mathrm{d} \tau \mathrm{d} s \\
& +\int_{t_{0}}^{t} \sum_{i=1}^{I} \bar{c}_{i}(s) u_{1}^{\alpha_{i}}(s) \mathrm{e}^{-\alpha_{i} \mu\left(s-t_{0}\right)} \int_{t_{0}}^{s} \sum_{l=1}^{L} \bar{v}_{l}(\theta) \int_{t_{0}}^{\theta} \bar{w}_{l}(\tau) u_{1}(\tau) \mathrm{e}^{-\mu\left(\tau-t_{0}\right)} \mathrm{d} \tau \mathrm{d} \theta \mathrm{d} s \\
& +\int_{t_{0}}^{t} \sum_{i=1}^{I} \bar{d}_{i}(s) u_{2}^{\alpha_{i}}(s) \int_{t_{0}}^{s} \sum_{l=1}^{L} \bar{x}_{l}(\theta) \int_{t_{0}}^{\theta} \bar{y}_{l}(\tau) u_{2}(\tau) \mathrm{d} \tau \mathrm{d} \theta \mathrm{d} s \\
& +\int_{t_{0}}^{t} \sum_{i=1}^{I} \bar{c}_{i}(s) u_{1}^{\alpha_{i}}(s) \mathrm{e}^{-\alpha_{i} \mu\left(s-t_{0}\right)} \int_{t_{0}}^{s} \sum_{m=1}^{M} \bar{A}_{m}(\theta) \int_{t_{0}}^{\theta} \bar{B}_{m}(\tau) u_{1}^{\gamma_{m}+1}(\tau) \mathrm{e}^{-\left(\gamma_{m}+1\right) \mu\left(\tau-t_{0}\right)} \mathrm{d} \tau \mathrm{d} \theta \mathrm{d} s \\
& +\int_{t_{0}}^{t} \sum_{i=1}^{I} \bar{d}_{i}(s) u_{2}^{\alpha_{i}}(s) \int_{t_{0}}^{s} \sum_{m=1}^{M} \bar{D}_{m}(\theta) \int_{t_{0}}^{\theta} \bar{E}_{m}(\tau) u_{2}^{\gamma_{m}+1}(\tau) \mathrm{d} \tau \mathrm{d} \theta \mathrm{d} s
\end{aligned}
$$

where: $u_{1}(t), u_{2}(t), a(t), \bar{a}(t), b(t), \bar{b}(t), c_{i}(t), \bar{c}_{i}(t), d_{i}(t), \bar{d}_{i}(t)(i=1,2, \cdots, I)$, $e_{j}(t), \bar{e}_{j}(t), f_{j}(t), \bar{f}_{j}(t), g_{j}(t), \bar{g}_{j}(t), h_{j}(t), \bar{h}_{j}(t)(j=1,2, \cdots, J)$, $o_{k}(t), \bar{o}_{k}(t), p_{k}(t), \bar{p}_{k}(t), q_{k}(t), \bar{q}_{k}(t), r_{k}(t), \bar{r}_{k}(t)(k=1,2, \cdots, K)$, $v_{l}(t), \bar{v}_{l}(t), w_{l}(t), \bar{w}_{l}(t), x_{l}(t), \bar{x}_{l}(t), y_{l}(t), \bar{y}_{l}(t)(l=1,2, \cdots, L)$, $A_{m}(t), \bar{A}_{m}(t), B_{m}(t), \bar{B}_{m}(t), D_{m}(t), \bar{D}_{m}(t), E_{m}(t), \bar{E}_{m}(t)(m=1,2, \cdots, M)$ are non-negative continuous function on $R_{+}$, and: $\alpha_{i}(i=1,2, \cdots, I)$, $\beta_{k}(k=1,2, \cdots, K), \quad \gamma_{m}(m=1,2, \cdots, M)$ are all constants greater than 1 , and $1 \leq \alpha_{1} \leq \alpha_{2} \leq \cdots \leq \alpha_{I}, 1 \leq \beta_{1} \leq \beta_{2} \leq \cdots \leq \beta_{K}, 1 \leq \gamma_{1} \leq \gamma_{2} \leq \cdots \leq \gamma_{M}$ set: $\bar{\alpha}=\max \left(\alpha_{I}, \beta_{K}, \gamma_{M}\right), \underline{\alpha}=\min \left(\alpha_{1}, \beta_{1}, \gamma_{1}\right)$

iii) let: $k=k_{1}+k_{2}$

$$
\begin{gathered}
F(t)=\max \{a(t)+\bar{a}(t), b(t)+\bar{b}(t)\}, \quad G_{i}(t)=\max \left\{c_{i}(t)+\bar{c}_{i}(t), d_{i}(t)+\bar{d}_{i}(t)\right\}, \\
\overline{\bar{e}}_{j}(t)=\max \left\{e_{j}(t), \bar{e}_{j}(t)\right\}, \overline{\bar{g}}_{j}(t)=\max \left\{g_{j}(t), \bar{g}_{j}(t)\right\},
\end{gathered}
$$




$$
\begin{gathered}
H_{j}(t)=\max \left\{\overline{\bar{e}}_{j}(t), \overline{\bar{g}}_{j}(t)\right\}, \quad N_{j}(t)=\max \left\{f_{j}(t)+\bar{f}_{j}(t), h_{j}(t)+\bar{h}_{j}(t)\right\}, \\
\quad \overline{\bar{o}}_{k}(t)=\max \left\{o_{k}(t), \bar{o}_{k}(t)\right\}, \quad \overline{\bar{q}}_{k}(t)=\max \left\{q_{k}(t), \bar{q}_{k}(t)\right\}, \\
Q_{k}(t)=\max \left\{\overline{\bar{o}}_{k}(t), \overline{\bar{q}}_{k}(t)\right\}, \quad R_{k}(t)=\max \left\{p_{k}(t)+\bar{p}_{k}(t), r_{k}(t)+\bar{r}_{k}(t)\right\}, \\
\quad \overline{\bar{v}}_{l}(t)=\max \left\{v_{l}(t), \bar{v}_{l}(t)\right\}, \quad \overline{\bar{x}}_{l}(t)=\max \left\{x_{l}(t), \bar{x}_{l}(t)\right\}, \\
T_{l}(t)=\max \left\{\overline{\bar{v}}_{l}(t), \overline{\bar{x}}_{l}(t)\right\}, \quad W_{l}(t)=\max \left\{w_{l}(t)+\bar{w}_{l}(t), y_{l}(t)+\bar{y}_{l}(t)\right\}, \\
\overline{\bar{A}}_{m}(t)=\max \left\{A_{m}(t), \bar{A}_{m}(t)\right\}, \quad \overline{\bar{D}}_{m}(t)=\max \left\{D_{m}(t), \bar{D}_{m}(t)\right\}, \\
Y_{m}(t)=\max \left\{\overline{\bar{A}}_{m}(t), \overline{\bar{D}}_{m}(t)\right\}, \quad Z_{m}(t)=\max \left\{B_{m}(t)+\bar{B}_{m}(t), E_{m}(t)+\bar{E}_{m}(t)\right\},
\end{gathered}
$$

iv) Let:

$$
\begin{aligned}
& \Delta(t)=F(t)+\sum_{i=1}^{I} G_{i}(t)+\sum_{j=1}^{J} H_{j}(t) \int_{t_{0}}^{t} N_{j}(s) \mathrm{d} s+2 \sum_{k=1}^{K} Q_{k}(t) \int_{t_{0}}^{t} R_{k}(s) \mathrm{d} s \\
& +\sum_{i=1}^{I} G_{i}(t) \int_{t_{0}}^{t} \sum_{l=1}^{L} T_{l}(s) \int_{t_{0}}^{s} W_{l}(\sigma) \mathrm{d} \sigma \mathrm{d} s+\sum_{i=1}^{I} G_{i}(t) \int_{t_{0}}^{t} \sum_{m=1}^{M} Y_{m}(s) \int_{t_{0}}^{s} Z_{m}(\sigma) \mathrm{d} \sigma \mathrm{d} s \\
& \Phi(t)=\sum_{i=1}^{I} G_{i}(t)+\sum_{k=1}^{K} Q_{k}(t) \int_{t_{0}}^{t} R_{k}(s) \mathrm{d} s+\sum_{i=1}^{I} G_{i}(t) \int_{t_{0}}^{t} \sum_{l=1}^{L} T_{l}(s) \int_{t_{0}}^{s} W_{l}(\sigma) \mathrm{d} \sigma \mathrm{d} s \\
& +2 \sum_{i=1}^{I} G_{i}(t) \int_{t_{0}}^{t} \sum_{m=1}^{M} Y_{m}(s) \int_{t_{0}}^{s} Z_{m}(\sigma) \mathrm{d} \sigma \mathrm{d} s \\
& \Gamma(t)=\int_{t_{0}}^{t} \sum_{m=1}^{M} Y_{m}(s) \int_{t_{0}}^{s} Z_{m}(\sigma) \mathrm{d} \sigma \mathrm{d} s \\
& \Lambda_{1}(t)=1-\bar{\alpha} c^{\bar{\alpha}} \int_{t_{0}}^{t}[\Phi(\tau)+\Gamma(\tau)] \exp \left(\bar{\alpha} \int_{t_{0}}^{\tau} \Delta(\sigma) \mathrm{d} \sigma\right) \mathrm{d} \tau \\
& \Pi(t)=F(t)+\sum_{j=1}^{J} H_{j}(t) \int_{t_{0}}^{t} N_{j}(t) \mathrm{d} t
\end{aligned}
$$

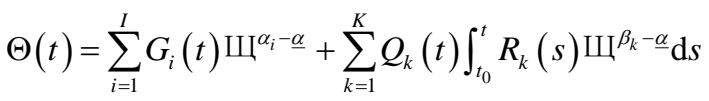

$$
\begin{aligned}
& +\sum_{i=1}^{I} G_{i}(t) щ^{\alpha_{i}-\underline{\alpha}} \int_{t_{0}}^{t} \sum_{l=1}^{L} T_{l}(s) \int_{t_{0}}^{s} W_{l}(\sigma) \mathrm{d} \sigma \\
& \Sigma(t)=\sum_{m=1}^{M} Y_{m}(t) \int_{t_{0}}^{t} Z_{m}(s){\Psi^{\gamma_{m}}-\underline{\alpha}}^{\mathrm{d}} s \\
& \Lambda_{2}(t)=1-\underline{\alpha} c^{\underline{\alpha}} \int_{t_{0}}^{t}[\Theta(\tau)+\Sigma(\tau)] \exp \left(\underline{\alpha} \int_{t_{0}}^{\tau} \Pi(\sigma) \mathrm{d} \sigma\right) \mathrm{d} \tau
\end{aligned}
$$

And $\int_{t_{0}}^{+\infty} \Delta(s) \mathrm{d} s<+\infty$,

$$
\begin{aligned}
& \Lambda_{1}(t)>0,\left[1-(\bar{\alpha}-1) c^{\bar{\alpha}} \int_{t_{0}}^{t} \Phi(s) \Lambda_{1}^{-\frac{1}{\bar{\alpha}}}(s) \exp \left(\bar{\alpha} \int_{t_{0}}^{s} \Delta(\tau) \mathrm{d} \tau\right) \mathrm{d} s\right]>0 \\
& \Lambda_{2}(t)>0,\left[1-(\underline{\alpha}-1) c^{\underline{\alpha}} \int_{t_{0}}^{t} \Theta(s) \Lambda_{2}^{-\frac{1}{\underline{\alpha}}}(s) \exp \left(\underline{\alpha} \int_{t_{0}}^{s} \Sigma(\tau) \mathrm{d} \tau\right) \mathrm{d} s\right]>0
\end{aligned}
$$

v) Assume: 
$\Omega(t) \leq k \exp \left(\int_{t_{0}}^{t} \Pi(s) \mathrm{d} s\right) \cdot\left[1-(\underline{\alpha}-1) k^{\underline{\alpha}} \int_{t_{0}}^{t} \Theta(s) \Lambda_{2}^{-\frac{1}{\alpha}}(s) \exp \left(\underline{\alpha} \int_{t_{0}}^{s} \Pi(\tau) \mathrm{d} \tau\right) \mathrm{d} s\right]^{-\frac{1}{\underline{\alpha}-1}}$ then: $u_{1}(t) \leq \Omega(t) \mathrm{e}^{\mu\left(t-t_{0}\right)}, \quad u_{2}(t) \leq \Omega(t)$.

\section{Main Results}

Consider the following system

$$
\left\{\begin{aligned}
\frac{\mathrm{d} y}{\mathrm{~d} t}= & B(t) y+C(t) z \\
& +Y\left(s, y(s), z(s), \int_{0}^{t} h_{1}(s, y(s), z(s), y(s-\tau), \dot{z}(s-\tau)) \mathrm{d} s\right) \\
\frac{\mathrm{d} z}{\mathrm{~d} t}= & D(t) y+E(t) z \\
& +Z\left(s, y(s), z(s), \int_{0}^{t} h_{2}(s, y(s), z(s), y(s-\tau), \dot{z}(s-\tau)) \mathrm{d} s\right)
\end{aligned}\right.
$$

where $\tau \geq 0$ is a constant, initial condition is:

$$
x(t)=\phi(t), \dot{x}(t)=\dot{\phi}(t), t_{0}-\tau \leq t \leq t_{0},
$$

$B(t)$ is an $m \times m$ matrix,

$Y\left(s, y(s), z(s), \int_{0}^{t} h_{1}(s, y(s), z(s), y(s-\tau), \dot{z}(s-\tau)) \mathrm{d} s\right)$ is an $m \times 1$ matrix,

$z\left(s, y(s), z(s), \int_{0}^{t} h_{2}(s, y(s), z(s), y(s-\tau), \dot{z}(s-\tau)) \mathrm{d} s\right)$ is an $(n-m) \times 1 \mathrm{ma}-$ trix, they are all continuous for $t \in I$ and satisfy the condition of existence and uniqueness theorem.

Set $Y(t, s)$ and $Z(t, s)$ satisfied:

$$
\begin{aligned}
& \left\{\begin{array}{l}
\frac{\partial Y(t, s)}{\partial t}=B(t) Y(t, s), \\
Y(s, s)=I
\end{array}\right. \\
& \left\{\begin{array}{l}
\frac{\partial Z(t, s)}{\partial t}=E(t) Z(t, s) \\
Z(s, s)=I
\end{array}\right.
\end{aligned}
$$

Theorem If (2) satisfies the following conditions:

i) $\|Y(t, s)\| \leq m_{1} \mathrm{e}^{-\lambda(t-s)},\|Z(t, s)\| \leq m_{2}$;

$$
\begin{aligned}
&\left\|Y\left(t, y, z, y(t-\tau), z(t-\tau), \int_{0}^{t} h_{1}(s, y, z, y(s-\tau), \dot{z}(s-\tau)) \mathrm{d} s\right)\right\| \\
& \leq a(t)(\|y(t-\delta(t))\|+\|\dot{y}(t-\delta(t))\|) \\
&\text { ii }) \quad+b(t)(\|z(t-\delta(t))\|+\|\dot{z}(t-\delta(t))\|) \mathrm{e}^{-\varepsilon\left(t-t_{0}\right)} \\
& \quad+\sum_{i=1}^{I} c_{i}(t)\left(\|y(t-\delta(t))\|^{\alpha_{i}+1}+\|\dot{y}(t-\delta(t))\|^{\alpha_{i}+1}\right) \mathrm{e}^{\alpha_{i} \varepsilon\left(t-t_{0}\right)} \\
& \quad+\sum_{i=1}^{I} d_{i}(t)\left(\|z(t-\delta(t))\|^{\alpha_{i}+1}+\|\dot{z}(t-\delta(t))\|^{\alpha_{i}+1}\right) \mathrm{e}^{-\varepsilon\left(t-t_{0}\right)}
\end{aligned}
$$




$$
\begin{aligned}
& +\sum_{j=1}^{J} e_{j}(t) \int_{t_{0}}^{t} f_{j}(s)(\|y(s-\delta(s))\|+\|\dot{y}(s-\delta(s))\|) \mathrm{d} s \\
& +\sum_{j=1}^{J} g_{j}(t) \int_{t_{0}}^{t} h_{j}(s)(\|z(s-\delta(s))\|+\|\dot{z}(s-\delta(s))\|) \mathrm{e}^{-\varepsilon\left(s-t_{0}\right)} \mathrm{d} s \\
& +\sum_{k=1}^{K} o_{k}(t) \int_{t_{0}}^{t} p_{k}(s)\left(\|y(s-\delta(s))\|^{\beta_{k}+1}+\|\dot{y}(s-\delta(s))\|^{\beta_{k}+1}\right) \mathrm{e}^{\beta_{k} \varepsilon\left(s-t_{0}\right)} \mathrm{d} s \\
& +\sum_{k=1}^{K} q_{k}(t) \int_{t_{0}}^{t} r_{k}(s)\left(\|z(s-\delta(s))\|^{\beta_{k}+1}+\|\dot{z}(s-\delta(s))\|^{\beta_{k}+1}\right) \mathrm{e}^{-\varepsilon\left(s-t_{1}\right)} \mathrm{d} s \\
& +\sum_{i=1}^{I} c_{i}(t)\left(\|y(t-\delta(t))\|^{\alpha_{i}}+\|\dot{y}(t-\delta(t))\|^{\alpha_{i}}\right) \mathrm{e}^{\alpha_{i} \varepsilon\left(t-t_{0}\right)} \\
& \cdot \int_{t_{0}}^{t} \sum_{l=1}^{L} v_{l}(s) \int_{t_{0}}^{s} w_{l}(\theta)(\|y(\theta-\delta(\theta))\|+\|\dot{y}(\theta-\delta(\theta))\|) \mathrm{d} \theta \mathrm{d} s \\
& +\sum_{i=1}^{I} d_{i}(t)\left(\|z(t-\delta(t))\|^{\alpha_{i}}+\|\dot{z}(t-\delta(t))\|^{\alpha_{i}}\right) \\
& \cdot \int_{t_{0}}^{t} \sum_{l=1}^{L} x_{l}(s) \int_{t_{0}}^{s} y_{l}(\theta)(\|z(\theta-\delta(\theta))\|+\|\dot{z}(\theta-\delta(\theta))\|) \mathrm{e}^{-\varepsilon\left(\theta-t_{0}\right)} \mathrm{d} \theta \mathrm{d} s \\
& +\sum_{i=1}^{I} c_{i}(t)\left(\|y(t-\delta(t))\|^{\alpha_{i}}+\|\dot{y}(t-\delta(t))\|^{\alpha_{i}}\right) \mathrm{e}^{\alpha_{i} \varepsilon\left(t-t_{0}\right)} \\
& \cdot \int_{t_{0}}^{t} \sum_{m=1}^{M} A_{m}(s) \int_{t_{0}}^{s} B_{m}(\theta)\left(\|y(\theta-\delta(\theta))\|^{\gamma_{m}+1}+\|\dot{y}(\theta-\delta(\theta))\|^{\gamma_{m}+1}\right) \mathrm{e}^{\gamma_{m} \varepsilon\left(\theta-t_{0}\right)} \mathrm{d} \theta \mathrm{d} s \\
& +\sum_{i=1}^{I} d_{i}(t)\left(\|z(t-\delta(t))\|^{\alpha_{i}}+\|\dot{z}(t-\delta(t))\|^{\alpha_{i}}\right) \\
& \cdot \int_{t_{0}}^{t} \sum_{m=1}^{M} D_{m}(s) \int_{t_{0}}^{s} E_{m}(\theta)\left(\|z(\theta-\delta(\theta))\|^{\gamma_{m}+1}+\|\dot{z}(\theta-\delta(\theta))\|^{\gamma_{m}+1}\right) \mathrm{e}^{-\varepsilon\left(\theta-t_{0}\right)} \mathrm{d} \theta \mathrm{d} s \\
& \left\|Z\left(t, y, z, y(t-\tau), z(t-\tau), \int_{0}^{t} h_{2}(s, y, z, y(s-\tau), \dot{z}(s-\tau)) \mathrm{d} s\right)\right\| \\
& \leq \bar{a}(t)(\|y(t-\delta(t))\|+\|\dot{y}(t-\delta(t))\|) \mathrm{e}^{\varepsilon\left(t-t_{0}\right)} \\
& \text { iv) } \quad+\bar{b}(t)(\|z(t-\delta(t))\|+\|\dot{z}(t-\delta(t))\|) \\
& +\sum_{i=1}^{I} \bar{c}_{i}(t)\left(\|y(t-\delta(t))\|^{\alpha_{i}+1}+\|\dot{y}(t-\delta(t))\|^{\alpha_{i}+1}\right) \mathrm{e}^{\left(\alpha_{i}+1\right) \varepsilon\left(t-t_{0}\right)} \\
& +\sum_{i=1}^{I} \bar{d}_{i}(t)\left(\|z(t-\delta(t))\|^{\alpha_{i}+1}+\|\dot{z}(t-\delta(t))\|^{\alpha_{i}+1}\right) \\
& +\sum_{j=1}^{J} \bar{e}_{j}(t) \int_{t_{0}}^{t} \bar{f}_{j}(s)(\|y(s-\delta(s))\|+\|\dot{y}(s-\delta(s))\|) \mathrm{e}^{\varepsilon\left(s-t_{0}\right)} \mathrm{d} s \\
& +\sum_{j=1}^{J} \bar{g}_{j}(t) \int_{t_{0}}^{t} \bar{h}_{j}(s)(\|z(s-\delta(s))\|+\|\dot{z}(s-\delta(s))\|) \mathrm{d} s \\
& +\sum_{k=1}^{K} \bar{o}_{k}(t) \int_{t_{0}}^{t} \bar{p}_{k}(s)\left(\|y(s-\delta(s))\|^{\beta_{k}+1}+\|\dot{y}(s-\delta(s))\|^{\beta_{k}+1}\right) \mathrm{e}^{\left(\beta_{k}+1\right) \varepsilon\left(s-t_{0}\right)} \mathrm{d} s \\
& +\sum_{k=1}^{K} \bar{q}_{k}(t) \int_{t_{0}}^{t} \bar{r}_{k}(s)\left(\|z(s-\delta(s))\|^{\beta_{k}+1}+\|\dot{z}(s-\delta(s))\|^{\beta_{k}+1}\right) \mathrm{d} s
\end{aligned}
$$




$$
\begin{gathered}
+\sum_{i=1}^{I} \bar{c}_{i}(t)\left(\|y(t-\delta(t))\|^{\alpha_{i}}+\|\dot{y}(t-\delta(t))\|^{\alpha_{i}}\right) \mathrm{e}^{\alpha_{i} \varepsilon\left(t-t_{0}\right)} \\
\cdot \int_{t_{0}}^{t} \sum_{l=1}^{L} v_{l}(s) \int_{t_{0}}^{s} w_{l}(\theta)(\|y(\theta-\delta(\theta))\|+\|\dot{y}(\theta-\delta(\theta))\|) \mathrm{e}^{\varepsilon\left(\theta-t_{0}\right)} \mathrm{d} \theta \mathrm{d} s \\
+\sum_{i=1}^{I} \bar{d}_{i}(t)\left(\|z(t-\delta(t))\|^{\alpha_{i}}+\|\dot{z}(t-\delta(t))\|^{\alpha_{i}}\right) \\
\cdot \int_{t_{0}}^{t} \sum_{l=1}^{L} \bar{x}_{l}(s) \int_{t_{0}}^{s} \bar{y}_{l}(\theta)(\|z(\theta-\delta(\theta))\|+\|\dot{z}(\theta-\delta(\theta))\|) \mathrm{d} \theta \mathrm{d} s \\
+\sum_{i=1}^{I} \bar{c}_{i}(t)\left(\|y(t-\delta(t))\|^{\alpha_{i}}+\left.\|\dot{y}(t-\delta(t))\|\right|^{\alpha_{i}}\right) \mathrm{e}^{\alpha_{i} \varepsilon\left(t-t_{0}\right)} \\
\cdot \int_{t_{0}}^{t} \sum_{m=1}^{M} \bar{A}_{m}(s) \int_{t_{0}}^{s} \bar{B}_{m}(\theta)\left(\|y(\theta-\delta(\theta))\|^{\gamma_{m}+1}+\|\dot{y}(\theta-\delta(\theta))\|^{\gamma_{m}+1}\right) \mathrm{e}^{\left(\gamma_{m}+1\right) \varepsilon\left(\theta-t_{0}\right)} \mathrm{d} \theta \mathrm{d} s \\
+\sum_{i=1}^{I} \bar{d}_{i}(t)\left(\|z(t-\delta(t))\|^{\alpha_{i}}+\|\dot{z}(t-\delta(t))\|^{\alpha_{i}}\right) \\
\cdot \int_{t_{0}}^{t} \sum_{m=1}^{M} \bar{D}_{m}(s) \int_{t_{0}}^{s} \bar{E}_{m}(\theta)\left(\|z(\theta-\delta(\theta))\|^{\gamma_{m}+1}+\|\dot{z}(\theta-\delta(\theta))\|^{\gamma_{m}+1}\right) \mathrm{d} \theta \mathrm{d} s
\end{gathered}
$$

where: $a(t), \bar{a}(t), b(t), \bar{b}(t), c_{i}(t), \bar{c}_{i}(t), d_{i}(t), \bar{d}_{i}(t)(i=1,2, \cdots, I)$, $e_{j}(t), \bar{e}_{j}(t), f_{j}(t), \bar{f}_{j}(t), g_{j}(t), \bar{g}_{j}(t), h_{j}(t), \bar{h}_{j}(t)(j=1,2, \cdots, J)$, $o_{k}(t), \bar{o}_{k}(t), p_{k}(t), \bar{p}_{k}(t), q_{k}(t), \bar{q}_{k}(t), r_{k}(t), \bar{r}_{k}(t)(k=1,2, \cdots, K)$, $v_{l}(t), \bar{v}_{l}(t), w_{l}(t), \bar{w}_{l}(t), x_{l}(t), \bar{x}_{l}(t), y_{l}(t), \bar{y}_{l}(t)(l=1,2, \cdots, L)$, $A_{m}(t), \bar{A}_{m}(t), B_{m}(t), \bar{B}_{m}(t), D_{m}(t), \bar{D}_{m}(t), E_{m}(t), \bar{E}_{m}(t)(m=1,2, \cdots, M) \quad$ are non-negative continuous monotonic non-increasing functions on $R_{+}$, and: $\alpha_{i}(i=1,2, \cdots, I), \quad \beta_{k}(k=1,2, \cdots, K), \quad \gamma_{m}(m=1,2, \cdots, M)$ are all constants greater than $1,1 \leq \alpha_{1} \leq \alpha_{2} \leq \cdots \leq \alpha_{I}, 1 \leq \beta_{1} \leq \beta_{2} \leq \cdots \leq \beta_{K}, 1 \leq \gamma_{1} \leq \gamma_{2} \leq \cdots \leq \gamma_{M}$ let: $\bar{\alpha}=\max \left(\alpha_{I}, \beta_{K}, \gamma_{M}\right), \underline{\alpha}=\min \left(\alpha_{1}, \beta_{1}, \gamma_{1}\right)$

iv) Set:

$$
\begin{gathered}
\Delta(t)=F(t)+\sum_{i=1}^{I} G_{i}(t)+\sum_{j=1}^{J} H_{j}(t) \int_{t_{0}}^{t} N_{j}(s) \mathrm{d} s+2 \sum_{k=1}^{K} Q_{k}(t) \int_{t_{0}}^{t} R_{k}(s) \mathrm{d} s \\
+\sum_{i=1}^{I} G_{i}(t) \int_{t_{0}}^{t} \sum_{l=1}^{L} T_{l}(s) \int_{t_{0}}^{s} W_{l}(\sigma) \mathrm{d} \sigma \mathrm{d} s+\sum_{i=1}^{I} G_{i}(t) \int_{t_{0}}^{t} \sum_{m=1}^{M} Y_{m}(s) \int_{t_{0}}^{s} Z_{m}(\sigma) \mathrm{d} \sigma \mathrm{d} s \\
\Phi(t)=\sum_{i=1}^{I} G_{i}(t)+\sum_{k=1}^{K} Q_{k}(t) \int_{t_{0}}^{t} R_{k}(s) \mathrm{d} s+\sum_{i=1}^{I} G_{i}(t) \int_{t_{0}}^{t} \sum_{l=1}^{L} T_{l}(s) \int_{t_{0}}^{s} W_{l}(\sigma) \mathrm{d} \sigma \mathrm{d} s \\
+2 \sum_{i=1}^{I} G_{i}(t) \int_{t_{0}}^{t} \sum_{m=1}^{M} Y_{m}(s) \int_{t_{0}}^{s} Z_{m}(\sigma) \mathrm{d} \sigma \mathrm{d} s \\
\Gamma(t)=\int_{t_{0}}^{t} \sum_{m=1}^{M} Y_{m}(s) \int_{t_{0}}^{s} Z_{m}(\sigma) \mathrm{d} \sigma \mathrm{d} s \\
\Lambda_{1}(t)=1-\bar{\alpha} c^{\bar{\alpha}} \int_{t_{0}}^{t}[\Phi(\tau)+\Gamma(\tau)] \exp \left(\bar{\alpha} \int_{t_{0}}^{\tau} \Delta(\sigma) \mathrm{d} \sigma\right) \mathrm{d} \tau \\
\Pi(t)=F(t)+\sum_{j=1}^{J} H_{j}(t) \int_{t_{0}}^{t} N_{j}(t) \mathrm{d} t \\
\Theta(t)=\sum_{i=1}^{I} G_{i}(t) \amalg^{\alpha_{i}-\underline{\alpha}}+\sum_{k=1}^{K} Q_{k}(t) \int_{t_{0}}^{t} R_{k}(s) \underline{\beta}^{\beta_{k}-\underline{\alpha}} \mathrm{d} s \\
+\sum_{i=1}^{I} G_{i}(t) \amalg^{\alpha_{i}-\underline{\alpha}} \int_{t_{0}}^{t} \sum_{l=1}^{L} T_{l}(s) \int_{t_{0}}^{s} W_{l}(\sigma) \mathrm{d} \sigma
\end{gathered}
$$




$$
\begin{gathered}
\Sigma(t)=\sum_{m=1}^{M} Y_{m}(t) \int_{t_{0}}^{t} Z_{m}(s) Щ^{\gamma_{m}-\underline{\alpha}} \mathrm{d} s \\
\Lambda_{2}(t)=1-\underline{\alpha} c^{\underline{\alpha}} \int_{t_{0}}^{t}[\Theta(\tau)+\Sigma(\tau)] \exp \left(\underline{\alpha} \int_{t_{0}}^{\tau} \Pi(\sigma) \mathrm{d} \sigma\right) \mathrm{d} \tau
\end{gathered}
$$

and $\int_{t_{0}}^{+\infty} \Delta(s) \mathrm{d} s<+\infty$

$$
\begin{aligned}
& \Lambda_{1}(t)>0,\left[1-(\bar{\alpha}-1) c^{\bar{\alpha}} \int_{t_{0}}^{t} \Phi(s) \Lambda_{1}^{-\frac{1}{\bar{\alpha}}}(s) \exp \left(\bar{\alpha} \int_{t_{0}}^{s} \Delta(\tau) \mathrm{d} \tau\right) \mathrm{d} s\right]>0 \\
& \Lambda_{2}(t)>0,\left[1-(\underline{\alpha}-1) c^{\underline{\alpha}} \int_{t_{0}}^{t} \Theta(s) \Lambda_{2}^{-\frac{1}{\alpha}}(s) \exp \left(\underline{\alpha} \int_{t_{0}}^{s} \Sigma(\tau) \mathrm{d} \tau\right) \mathrm{d} s\right]>0
\end{aligned}
$$

v) Set:

$\Omega(t)=k \exp \left(\int_{0}^{t} \Pi(s) \mathrm{d} s\right) \cdot\left[1-(\underline{\alpha}-1) k^{\underline{\alpha}} \int_{0}^{t} \Theta(s) \Lambda_{2}^{-\frac{1}{\alpha}}(s) \exp \left(\underline{\alpha} \int_{0}^{s} \Pi(\tau) \mathrm{d} \tau\right) \mathrm{d} s\right]^{-\frac{1}{\underline{\alpha}-1}}$ then:

1) when $\lambda>\varepsilon$, the trivial solution of (2) is $L S, G E_{q} E L A S$ with respect to $y$,

2) when $\lambda=\varepsilon$, the trivial solution of (2) is, GUELAS with respect to.

Proof Apply constant variation method to system (2), it can be deduced that:

$$
\begin{aligned}
& y(t)=Y\left(t, t_{0}\right) y_{0}+\int_{t_{0}}^{t} Y(t, s) F_{1}\left(s, x(s-\delta(s)), \int_{t_{0}}^{s} h_{1}(\tau, x(\tau-\delta(\tau))) \mathrm{d} \tau\right) \mathrm{d} s \\
& Z(t)=Z\left(t, t_{0}\right) z_{0}+\int_{t_{0}}^{t} Z(t, s) F_{2}\left(s, x(s-\delta(s)), \int_{t_{0}}^{s} h_{2}(\tau, x(\tau-\delta(\tau))) \mathrm{d} \tau\right) \mathrm{d} s
\end{aligned}
$$

By the condition of the theory, available from (3):

$$
\begin{aligned}
\|y(t)\| \leq & m_{1} \mathrm{e}^{-\lambda\left(t-t_{0}\right)}\left\|\varphi_{1}\right\|+\int_{t_{0}}^{t} m_{1} \mathrm{e}^{-\lambda(t-s)}\left\{a(s)\|y(s-\delta(s))\|+b(s)\|z(s-\delta(s))\| \mathrm{e}^{-\varepsilon\left(s-t_{0}\right)}\right. \\
& +\sum_{i=1}^{I} c_{i}(s)\|y(s-\delta(s))\|^{\alpha_{i}+1} \mathrm{e}^{\alpha_{i} \varepsilon\left(s-t_{0}\right)}+\sum_{i=1}^{I} d_{i}(s)\|z(s-\delta(s))\|^{\alpha_{i}+1} \mathrm{e}^{-\varepsilon\left(s-t_{0}\right)} \\
& +\sum_{j=1}^{J} e_{j}(s) \int_{t_{0}}^{s} f_{j}(\theta)\|y(\theta-\delta(\theta))\| \mathrm{d} \theta+\sum_{j=1}^{J} g_{j}(s) \int_{t_{0}}^{s} h_{j}(\theta)\|z(\theta-\delta(\theta))\| \mathrm{e}^{-\varepsilon\left(\theta-t_{0}\right)} \mathrm{d} \theta \\
& +\sum_{k=1}^{K} o_{k}(s) \int_{t_{0}}^{s} p_{k}(\theta)\|y(\theta-\delta(\theta))\|^{\beta_{k}+1} \mathrm{e}^{\beta_{k} \varepsilon\left(\theta-t_{0}\right)} \mathrm{d} \theta \\
& +\sum_{k=1}^{K} q_{k}(s) \int_{t_{0}}^{s} r_{k}(\theta)\|z(\theta-\delta(\theta))\|^{\beta_{k}+1} \mathrm{e}^{-\varepsilon\left(\theta-t_{0}\right)} \mathrm{d} \theta \\
& +\sum_{i=1}^{I} c_{i}(s)\|y(s-\delta(s))\|^{\alpha_{i}} \mathrm{e}^{\alpha_{i} \varepsilon\left(s-t_{0}\right)} \int_{t_{0}}^{s} \sum_{l=1}^{L} v_{l}(\sigma) \int_{t_{0}}^{\sigma} w_{l}(\theta)\|y(\theta-\delta(\theta))\| \mathrm{d} \theta \mathrm{d} \sigma \\
& +\sum_{i=1}^{I} d_{i}(s)\|z(s-\delta(s))\|^{\alpha_{i}} \int_{t_{0}}^{s} \sum_{l=1}^{L} x_{l}(\sigma) \int_{t_{0}}^{\sigma} y_{l}(\theta)\|z(\theta-\delta(\theta))\| \mathrm{e}^{-\varepsilon\left(\theta-t_{0}\right)} \mathrm{d} \theta \mathrm{d} \sigma \\
& +\sum_{i=1}^{I} c_{i}(s)\|y(s-\delta(s))\|^{\alpha_{i}} \mathrm{e}^{\alpha_{i} \varepsilon\left(s-t_{0}\right)} \int_{t_{0}}^{s} \sum_{m=1}^{M} A_{m}(\sigma) \int_{t_{0}}^{\sigma} B_{m}(\theta)\|y(\theta-\delta(\theta))\|^{\gamma_{m}+1} \mathrm{e}^{\gamma_{m} \varepsilon\left(\theta-t_{0}\right)} \mathrm{d} \theta \mathrm{d} v \\
& \left.+\sum_{i=1}^{I} d_{i}(s)\|z(s-\delta(s))\|^{\alpha_{i}} \int_{t_{0}}^{s} \sum_{m=1}^{M} D_{m}(\sigma) \int_{t_{0}}^{\sigma} E_{m}(\theta)\|z(\theta-\delta(\theta))\| \|^{\gamma_{m}+1} \mathrm{e}^{-\varepsilon\left(\theta-t_{0}\right)} \mathrm{d} \theta \mathrm{d} \sigma\right\} \mathrm{d} s
\end{aligned}
$$


however, set

$$
\begin{gathered}
u_{1}(t)=(\|y(t)\|+\|\dot{y}(t)\|) \mathrm{e}^{\lambda\left(t-t_{0}\right)}, \quad u_{2}(t)=\|z(t)\|+\|\dot{z}(t)\| \\
\varphi_{1}^{(1)}=\sup _{-\delta \leq t \leq 0}\left\|\varphi_{1}(t)\right\|, \quad \varphi_{2}^{(1)}=\sup _{-\delta \leq t \leq 0}\left\|\varphi_{1}(t)\right\|^{\alpha_{i}+1} \\
\varphi_{3}^{(1)}=\sup _{-\delta \leq t \leq 0}\left\|\varphi_{1}(t)\right\|^{\beta_{k}+1}, \varphi_{4}^{(1)}=\sup _{-\delta \leq t \leq 0}\left\|\varphi_{1}(t)\right\|^{\gamma_{m}+1} \\
\varphi^{(1)}=\max \left\{\varphi_{1}^{(1)}, \varphi_{2}^{(1)}, \varphi_{3}^{(1)}, \varphi_{4}^{(1)}, \varphi_{2}^{(1)} \varphi_{4}^{(1)}\right\} \\
\varphi_{1}^{(2)}=\sup _{-\delta \leq t \leq 0}\left\|\varphi_{2}(t)\right\|, \varphi_{2}^{(2)}=\sup _{-\delta \leq t \leq 0}\left\|\varphi_{2}(t)\right\|^{\alpha_{i}+1} \\
\varphi_{3}^{(2)}=\sup _{-\delta \leq t \leq 0}\left\|\varphi_{2}(t)\right\|^{\beta_{k}+1}, \varphi_{4}^{(2)}=\sup _{-\delta \leq t \leq 0}\left\|\varphi_{2}(t)\right\|^{\gamma_{m}+1} \\
\varphi^{(2)}=\max \left\{\varphi_{1}^{(2)}, \varphi_{2}^{(2)}, \varphi_{3}^{(2)}, \varphi_{4}^{(2)}, \varphi_{2}^{(2)} \varphi_{4}^{(2)}\right\}
\end{gathered}
$$

Set:

$$
\begin{aligned}
& \left\|u_{1}(t)\right\|= \begin{cases}\max \left\{\varphi_{1}^{(1)}, \max (u(\xi))\right\}, & 0 \leq \xi \leq t \\
\varphi_{1}^{(1)}, & -\delta \leq t \leq 0\end{cases} \\
& \left\|u_{2}(t)\right\|= \begin{cases}\max \left\{\varphi_{1}^{(2)}, \max (u(\xi))\right\}, & 0 \leq \xi \leq t \\
\varphi_{1}^{(2)}, & -\delta \leq t \leq 0\end{cases}
\end{aligned}
$$

Obviously, $\left\|u_{1}(t)\right\|,\left\|u_{2}(t)\right\|$ are monotonous, and defined by, $u_{1}(t), u_{2}(t)$, we have

$$
\begin{aligned}
& u_{1}(t-\delta(t)) \leq \varphi_{1}^{(1)}, \\
& u_{2}(t-\delta(t)) \leq \varphi_{1}^{(2)}
\end{aligned}
$$

So substituting (5) into (2) gives:

$u_{1}(t)$

$\leq \varphi^{(1)}+M_{2} \int_{t_{0}}^{t} a(s)\left\|u_{1}(s)\right\| \mathrm{d} s+M_{2} \int_{t_{0}}^{t} b(s)\left\|u_{2}(s)\right\| \mathrm{e}^{(\lambda-\varepsilon)\left(s-t_{0}\right)} \mathrm{d} s$

$+M_{2} \int_{t_{0}}^{t} \sum_{i=1}^{I} c_{i}(s)\left\|u_{1}(s)\right\|^{\alpha_{i}+1} \mathrm{e}^{-\alpha_{i}(\lambda-\varepsilon)\left(s-t_{0}\right)} \mathrm{d} s+M_{2} \int_{t_{0}}^{t} \sum_{i=1}^{I} d_{i}(s)\left\|u_{2}(s)\right\|^{\alpha_{i}+1} \mathrm{e}^{(\lambda-\varepsilon)\left(\theta-t_{0}\right)} \mathrm{d} s$

$+M_{2} \int_{t_{0}}^{t} \sum_{j=1}^{J} e_{j}(s) \int_{t_{0}}^{s} f_{j}(\theta)\left\|u_{1}(\theta)\right\| \mathrm{d} \theta \mathrm{d} s$

$+M_{2} \int_{t_{0}}^{t} \sum_{j=1}^{J} g_{j}(s) \int_{t_{0}}^{s} h_{j}(\theta)\left\|u_{2}(\theta)\right\| \mathrm{e}^{(\lambda-\varepsilon)\left(\theta-t_{0}\right)} \mathrm{d} \theta \mathrm{d} s$

$+M_{2} \int_{t_{0}}^{t} \sum_{k=1}^{K} o_{k}(s) \int_{t_{0}}^{s} p_{k}(\theta)\left\|u_{1}(\theta)\right\|^{\beta_{k}+1} \mathrm{e}^{-\beta_{k}(\lambda-\varepsilon)\left(\theta-t_{0}\right)} \mathrm{d} \theta \mathrm{d} s$ 


$$
\begin{aligned}
& +M_{2} \int_{t_{0}}^{t} \sum_{k=1}^{K} q_{k}(s) \int_{t_{0}}^{s} r_{k}(\theta)\left\|u_{2}(\theta)\right\|^{\beta_{k}+1} \mathrm{e}^{(\lambda-\varepsilon)\left(\theta-t_{0}\right)} \mathrm{d} \theta \mathrm{d} s \\
& +M_{2} \int_{t_{0}}^{t} \sum_{i=1}^{I} c_{i}(s)\left\|u_{1}(s)\right\|^{\alpha_{i}} \mathrm{e}^{-\alpha_{i}(\lambda-\varepsilon)\left(s-t_{0}\right)} \int_{t_{0}}^{s} \sum_{l=1}^{L} v_{l}(\sigma) \int_{t_{0}}^{\sigma} w_{l}(\theta)\left\|u_{1}(\theta)\right\| \mathrm{d} \theta \mathrm{d} \sigma \mathrm{d} s \\
& +M_{2} \int_{t_{0}}^{t} \sum_{i=1}^{I} d_{i}(s)\left\|u_{2}(s)\right\|^{\alpha_{i}} \int_{t_{0}}^{s} \sum_{l=1}^{L} x_{l}(\sigma) \int_{t_{0}}^{\sigma} y_{l}(\theta)\left\|u_{2}(\theta)\right\| \mathrm{e}^{(\lambda-\varepsilon)\left(\theta-t_{0}\right)} \mathrm{d} \theta \mathrm{d} \sigma \mathrm{d} s \\
& +M_{2} \int_{t_{0}}^{t} \sum_{i=1}^{I} c_{i}(s)\left\|u_{2}(s)\right\|^{\alpha_{i}} \mathrm{e}^{-\alpha_{i}(\lambda-\varepsilon)\left(s-t_{0}\right)} \int_{t_{0}}^{s} \sum_{m=1}^{M} A_{m}(\sigma) \int_{t_{0}}^{\sigma} B_{m}(\theta)\left\|u_{1}(\theta)\right\|^{\gamma_{m}+1} \mathrm{e}^{-\gamma_{m}(\lambda-\varepsilon)\left(\theta-t_{0}\right)} \mathrm{d} \theta \mathrm{d} \sigma \mathrm{d} s \\
& +M_{2} \int_{t_{0}}^{t} \sum_{i=1}^{I} d_{i}(s)\left\|u_{2}(s)\right\|^{\alpha_{i}} \int_{t_{0}}^{s} \sum_{m=1}^{M} D_{m}(\sigma) \int_{t_{0}}^{\sigma} E_{m}(\theta)\left\|u_{2}(\theta)\right\|^{\gamma_{m}+1} \mathrm{e}^{(\lambda-\varepsilon)\left(\theta-t_{0}\right)} \mathrm{d} \theta \mathrm{d} \sigma \mathrm{d} s
\end{aligned}
$$

Similarly available:

$$
\begin{aligned}
& u_{2}(t) \leq \varphi^{(2)}+M_{2} \int_{t_{0}}^{t} \bar{a}(s)\left\|u_{1}(s)\right\| \mathrm{e}^{-(\lambda-\varepsilon)\left(s-t_{0}\right)} \mathrm{d} s+M_{2} \int_{t_{0}}^{t} \bar{b}(s)\left\|u_{2}(s)\right\| \mathrm{d} s \\
& +M_{2} \int_{t_{0}}^{t} \sum_{i=1}^{I} \bar{c}_{i}(s)\left\|u_{1}(s)\right\|^{\alpha_{i}+1} \mathrm{e}^{-\left(\alpha_{i}+1\right)(\lambda-\varepsilon)\left(s-t_{0}\right)} \mathrm{d} s+M_{2} \int_{t_{0}}^{t} \sum_{i=1}^{I} \bar{d}_{i}(s)\left\|u_{2}(s)\right\|^{\alpha_{i}+1} \mathrm{~d} s \\
& +M_{2} \int_{t_{0}}^{t} \sum_{j=1}^{J} \bar{e}_{j}(s) \int_{t_{0}}^{s} \bar{f}_{j}(\theta)\left\|u_{1}(\theta)\right\| \mathrm{e}^{-(\lambda-\varepsilon)\left(\theta-t_{0}\right)} \mathrm{d} \theta \mathrm{d} s \\
& +M_{2} \int_{t_{0}}^{t} \sum_{j=1}^{J} \bar{g}_{j}(s) \int_{t_{0}}^{s} \bar{h}_{j}(\theta)\left\|u_{2}(\theta)\right\| \mathrm{d} \theta \mathrm{d} s \\
& +M_{2} \int_{t_{0}}^{t} \sum_{k=1}^{K} \bar{o}_{k}(s) \int_{t_{0}}^{s} \bar{p}_{k}(\theta)\left\|u_{1}(\theta)\right\|^{\beta_{k}+1} \mathrm{e}^{-\left(\beta_{k}+1\right)(\lambda-\varepsilon)\left(\theta-t_{0}\right)} \mathrm{d} \theta \mathrm{d} s \\
& +M_{2} \int_{t_{0}}^{t} \sum_{k=1}^{K} \bar{q}_{k}(s) \int_{t_{0}}^{s} \bar{r}_{k}(\theta)\left\|u_{2}(\theta)\right\|^{\beta_{k}+1} \mathrm{~d} \theta \mathrm{d} s \\
& +M_{2} \int_{t_{0}}^{t} \sum_{i=1}^{I} \bar{c}_{i}(s)\left\|u_{1}(s)\right\|^{\alpha_{i}} \mathrm{e}^{-\alpha_{i}(\lambda-\varepsilon)\left(s-t_{0}\right)} \int_{t_{0}}^{s} \sum_{l=1}^{L} \bar{v}_{l}(\sigma) \int_{t_{0}}^{\sigma} \bar{w}_{l}(\theta)\left\|u_{1}(\theta)\right\| \mathrm{e}^{-(\lambda-\varepsilon)\left(\theta-t_{0}\right)} \mathrm{d} \theta \mathrm{d} \sigma \mathrm{d} s \\
& +M_{2} \int_{t_{0}}^{t} \sum_{i=1}^{I} \bar{d}_{i}(s)\left\|u_{2}(s)\right\|^{\alpha_{i}} \int_{t_{0}}^{s} \sum_{l=1}^{L} \bar{x}_{l}(\sigma) \int_{t_{0}}^{\sigma} \bar{y}_{l}(\theta)\left\|u_{2}(\theta)\right\| \mathrm{d} \theta \mathrm{d} \sigma \mathrm{d} s \\
& +M_{2} \int_{t_{0}}^{t} \sum_{i=1}^{I} \bar{c}_{i}(s)\left\|u_{1}(s)\right\|^{\alpha_{i}} \mathrm{e}^{-\alpha_{i}(\lambda-\varepsilon)\left(s-t_{0}\right)} \int_{t_{0}}^{s} \sum_{m=1}^{M} \bar{A}_{m}(\sigma) \int_{t_{0}}^{\sigma} \bar{B}_{m}(\theta)\left\|u_{1}(\theta)\right\|^{\gamma_{m}+1} \mathrm{e}^{-\left(\gamma_{m}+1\right)(\lambda-\varepsilon)\left(\theta-t_{0}\right)} \mathrm{d} \theta \mathrm{d} \sigma \mathrm{d} s \\
& +M_{2} \int_{t_{0}}^{t} \sum_{i=1}^{I} \bar{d}_{i}(s)\left\|u_{2}(s)\right\|^{\alpha_{i}} \int_{t_{0}}^{s} \sum_{m=1}^{M} \bar{D}_{m}(\sigma) \int_{t_{0}}^{\sigma} \bar{E}_{m}(\theta)\left\|u_{2}(\theta)\right\|^{\gamma_{m}+1} \mathrm{~d} \theta \mathrm{d} \sigma \mathrm{d} s \\
& \text { where } M_{2}=\max \left\{M_{1}, M_{1}^{\alpha_{i}+1}, M_{1}^{\beta_{k}+1}, M_{1}^{\gamma_{m}+1}, M_{1}^{\alpha_{i}+\gamma_{m}+1}\right\} .
\end{aligned}
$$

So it can be obtained from Lemma: $u_{1}(t) \leq k \mathrm{e}^{(\lambda-\varepsilon)\left(t-t_{0}\right)} \Omega(t), u_{2}(t) \leq k \Omega(t)$ here $k=\mathrm{e}^{\lambda t_{0}} \phi, \phi=\max \left(\varphi^{(1)}, \varphi^{(2)}\right), \Omega(t)$ As the lemma states, then:

$$
\begin{gathered}
\|y(t)\|+\|\dot{y}(t)\| \leq M_{3} \varphi \mathrm{e}^{-\varepsilon\left(t-t_{0}\right)}(\lambda>\varepsilon),\|y(t)\|+\|\dot{y}(t)\| \leq M_{4} \varphi \mathrm{e}^{-\varepsilon\left(t-t_{0}\right)}(\lambda=\varepsilon) \\
\|z(t)\|+\|\dot{z}(t)\| \leq M_{5} \varphi
\end{gathered}
$$

here: $M_{3}=\mathrm{e}^{(\lambda-\varepsilon) t_{0}} \Omega(t) ; \quad M_{4}=\Omega(t) ; M_{5}=\mathrm{e}^{\lambda t_{0}} \Omega(t)$.

Notice the theorem conditions, we have $M_{1}$ is a constant that has nothing to do with $t_{0}, M_{2}$ and $M_{3}$ are constants that has nothing to do with $t_{0}$.

Therefore, when $\lambda>\varepsilon$, (6) means the trivial solution of (2) is LS, GE ELAS with respect to $y$, when $\lambda=\varepsilon$, (6) means the trivial solution of (2) is $L S$, GUELAS with respect to $y$. 
Note: The differential system discussed in this paper is the time-differential form of the ordinary differential system in [5] [6]. The time-differential system in [7] is generalized to a neutral system, and the Lipschitz stability in [8] is further extended to equi-exponential Lipschitz asymptotic stability and uniform exponential Lipschitz asymptotic stability and added global results

\section{Conclusion}

In this paper, we use the method of integral inequalities to establish double stability criteria. As a result, studying the partial stability of differential equations becomes more important. In addition, the partial stability of differential equations is widely used in science and technology.

\section{Conflicts of Interest}

The authors declare no conflicts of interest regarding the publication of this paper.

\section{References}

[1] Lyapunov, A.M. (1892) The General Problem of the Stability of Motion. Doctor dissertation, University of Moscow, Moscow.

[2] Li, Y.S. (1960) Basic Inequalities and Uniqueness of Solutions of Differential Equations. Journal of Jilin University, 1, 7-12.

[3] Si, L.G. (2005) A Note on a Class of Integral Inequalities. Journal of Inner Mongolia Normal University, 34, 127-128.

[4] Huo, R., Wang, X.L. and Si, L.G. (2009) A Generalization of a Class of Integral Inequality. Journal of Inner Mongolia Agricultral University (Natural Science Edition), 30, 267-273.

[5] Vorotnikov, V.I. (1999) On the Theory of Lyapunov Stablity in Critical Cases. Dokl. Akad. Nank., 367, 481-484.

[6] Vorotnikov, V.I. (1999) On Problems of Stablity with Respect to Some of the Variables. Prikl. Mat. Meth., 63, 736-745. https://doi.org/10.1016/S0021-8928(99)00088-X

[7] Wang, F. (2002) Stability of Differential Equations on Some Variables. Inner Mongolia Normal University, Hohhot.

[8] Si, L.G. (2006) The Stability of a Class of Nonlinear Differential Systems with Respect to Partial Variables. Journal of Inner Mongolia Normal University, 35, 1-6.

[9] Si, L.G. (1974) The Boundness and Stablity of Solutions for Nonlinear Neutral Differential Systems with Variable Delay. Acta Mathematica Sinica, 17, 197-204.

[10] Zhang, Y. (1994) K-Stablity of Nonlinear System with Time-Delay. Scientia Sinica Mathematica, 37, 247-255.

[11] Si, L.G. (2001) Differential Inequality and Differential Quation with Time-Delay, Inner Mongolia People's Press, Hohhot. 